\title{
PLANTAS DO GÊNERO Bauhinia: COMPOSIÇÃo QUÍMICA E POTENCIAL FARMACOLÓGICO
}

\author{
Karina Luize da Silva e Valdir Cechinel Filho* \\ Curso de Farmácia, CCS, Universidade do Vale do Itajaí, 88302-202 Itajaí - SC
}

Recebido em 11/12/00; aceito em 4/9/01

\begin{abstract}
PLANTS OF THE GENUS Bauhinia: CHEMICAL COMPOSITION AND PHARMACOLOGICAL POTENTIAL. Plants of the genus Bauhinia (Leguminosae) are widely distributed in most tropical countries and have been frequently used in folk medicine to treat several ailments, especially diabetes. In recent years, the interest in these plants has increased considerably in all world. This review shows the main chemical and pharmacological aspects of these plants. The studies carried out with the extracts and purified compounds from these plants support most of their reported therapeutic properties. It also gives an account of some compounds including terpenes, steroids and flavonoids that were isolated from the genus Bauhinia.
\end{abstract}

Keywords: Bauhinia; folk medicine; chemical composition; pharmacological effects.

\section{INTRODUÇÃO}

As plantas medicinais e seus derivados consistiram durante muito tempo a base da terapêutica e, atualmente, cerca de $25 \%$ dos fármacos utilizados são de origem vegetal, enquanto $50 \%$ são de origem sintética, mas relacionados aos princípios isolados de plantas medicinais ${ }^{1,2}$. Isto deve-se, em parte, à grande variedade de espécies (250-500 mil) de plantas existentes na flora mundial, muitas com importantes propriedades terapêuticas ${ }^{3}$. Nos últimos anos, tem-se verificado um grande aumento nos estudos que comprovam o que se conhece empiricamente, visto que a medicina popular é rica em exemplos de plantas utilizadas para diversos fins, que substituem, muitas vezes, a prescrição médica ${ }^{4-8}$. Acredita-se que cerca de $80 \%$ da população mundial use as plantas como primeiro recurso terapêutico ${ }^{9,10}$.

Entre as inúmeras espécies vegetais de interesse medicinal, encontram-se as plantas do gênero Bauhinia, pertencentes à família Leguminosae, as quais são encontradas principalmente nas áreas tropicais do planeta, compreendendo aproximadamente 300 espécies. Muitas destas plantas são usadas como remédio na medicina popular em várias regiões do mundo, incluindo a África, Ásia e América Central e do $\mathrm{Sul}^{11}$. Os estudos fitoquímicos e farmacológicos realizados com estas plantas indicam que as mesmas são constituídas principalmente de glicosídios esteroídicos, triterpenos, lactonas e flavonóides ${ }^{7,12}$.

No Brasil, as plantas do gênero Bauhinia são conhecidas como "Pata-de-vaca" ou "Unha-de-boi". As folhas, caules e raízes das espécies de Bauhinia, especialmente B. manca, B. rufescens, $B$. forficata, $B$. cheitantha e B. splendens, são amplamente utilizadas no Brasil e em outros países em forma de chás e outras preparações fitoterápicas para o tratamento de várias enfermidades, principalmente infecções, processos dolorosos e diabetes ${ }^{11,13,14}$.

$\mathrm{O}$ presente artigo tem como objetivo revisar os principais aspectos químicos e farmacológicos ou biológicos do gênero Bauhinia, descrevendo, quando possível e conhecido, os compostos isolados de cada espécie desse gênero, bem como a ação farmacológica ou biológica dessas substâncias.

* Núcleo de Investigações Químico-Farmacêuticas (NIQFAR)

e-mail: cechinel@univali.br

\section{COMPOSTOS ISOLADOS DAS PLANTAS DO GÊNERO Bauhinia}

Algumas espécies de plantas do gênero Bauhinia foram e estão sendo estudadas fitoquimica e farmacologicamente e, com isto, muitos compostos foram isolados e identificados. Assim, diferentes classes de compostos orgânicos de interesse medicinal existentes nessas espécies foram relatados, incluindo lactonas, flavonóides, terpenóides, esteróides, triterpenos, taninos e quinonas (Tabela 1).

Dentre as espécies mais estudadas fitoquimicamente, podemos citar B. manca, B. candicans, B. uruguayensis, B. purpurea, B. forficata e $B$. splendens. Neste gênero, como pode-se notar na Tabela 1, encontra-se uma maior variedade de compostos esteroídicos, terpenóides e flavonóides. Porém, apesar de muitos compostos serem conhecidos, pouco se conhece sobre a atividade farmacológica da maioria das substâncias isoladas do gênero Bauhinia.

Existem outras espécies de Bauhinia conhecidas e muito usadas na medicina popular, como a B. monandra, B. holophylla e B. rufa $\mathrm{f}^{37}$, porém não foram encontrados estudos sobre a constituição química e atividade farmacológica destas espécies.

Algumas estruturas moleculares de compostos novos ou raros isolados deste gênero serão mostradas no decorrer do artigo.

\section{ASPECTOS FARMACOLÓGICOS DO GÊNERO Bauhinia}

\section{Espécies com ação hipoglicemiante}

Como já mencionado anteriormente, pouco se conhece a respeito da atividade farmacológica das plantas do gênero Bauhinia, porém, este gênero é mais freqüentemente estudado quanto à sua possível ação hipoglicemiante, uma vez que na medicina popular estas plantas são usadas para o tratamento de diabetes ${ }^{13,14}$.

Uma das plantas que tem sido estudada quanto a atividade hipoglicemiante é a $B$. divaricata, conhecida no México como "pezunã de vaca", cuja decocção obtida das folhas apresentou moderado efeito hipoglicêmico em coelhos normais ${ }^{38}$. A infusão a $20 \%$ das folhas secas de B. candicans reduziu em $39 \%$ a glicemia induzida pela aloxana ${ }^{39}$, estando este efeito relacionado à presença do alcalóide trigonelina $^{15,40,41}$. B. forficata é a espécie que apresenta maior número de estudos quanto à atividade hipoglicemiante, sendo considerada, muitas vezes pela comunidade rural, como pata-de-vaca verdadeira, sendo muito usada na forma de chás e outras preparações 
Tabela 1. Principais compostos isolados de plantas do gênero Bauhinia

\begin{tabular}{|c|c|c|c|}
\hline ESPÉCIE & CLASSE & COMPOSTO & REF. \\
\hline \multirow[t]{8}{*}{ B.candicans } & Esteróides & Sitosterol ; Campesterol; Estigmasterol; Colesterol; & 15,16 \\
\hline & & Estigmasta-3,5-dieno-7-ona; Sitosterol 3-O- $\beta$ - glucosídio; & \\
\hline & & Sitosterol 3-O- $\alpha$-D-xilurono-furanosídio & \\
\hline & Flavonóides & Kaempferol 3-O- $\beta$-rutinosídio; Kaempferol 3-O- $\beta$-rutinosídio & \\
\hline & & 7-O- $\alpha$-rhamno-piranosídio & \\
\hline & Alcalóides & Trigonelina & \\
\hline & Álcoois & Triacontanol & \\
\hline & Poliálcoois & 3-O-metil-D-inositol (D-pinitol) & \\
\hline \multirow[t]{2}{*}{ B. championii } & Benzenóides & Ácido gálico & 17 \\
\hline & Glicosídios & Bauhinina & \\
\hline \multirow[t]{3}{*}{ B. forficata } & Flavonóides & Kaempferitrina; & 18 \\
\hline & & Kaempferol-3- $O$ - $\alpha$-Diraminosídio & 19 \\
\hline & Esteróides & Sitosterol & 18 \\
\hline \multirow[t]{3}{*}{ B.guianensis } & Esteróides & Sitosterol; Estigmasterol & 20 \\
\hline & Flavonóides & 4-hidroxi-7-metoxiflavona & \\
\hline & Quinonas & Lapachol; Di-hidro- $\alpha$-lapachona & \\
\hline
\end{tabular}

B. manca Esteróides

Sitosterol; Sitosterol-3-O- $\beta$-D-glucosídio

Estigmasta-4-eno-3-ona; Estigmasta-4-eno-3,6-diona

Benzenóides

Ácido cinâmico; cinnamoil- $\beta$-D-glucose; Éster metílico do ácido (E)-4-hidroxi-cinâmico; Éster metílico do ácido (E)-4hidróxi-3-metoxicinâmico; Ácido gálico, Galato de metila; Éster metílico do Ácido 4-hidroxi-2-metoxibenzóico; Éster metílico do Ácido 4-hidroxi-3-metoxibenzóico; Éster metílico do Ácido 3,4-dihidroxibenzóico; ๗-Hidroxipropioguaiacona; Siringaresinol;

\section{$(7 S, 8 R, 8 ' R)$-5,5-dimetoxilarici-resinol}

Flavonóides $\quad$ Apigenina; Chrisoeriol ; Luteolina 5,3-dimetoxi; Kaempferol; Isoliquiritigenina; Isoliquiritigenina 2-metoxi; Isoliquiritigenina 4metoxi; Echinatina; 2,4-di-hidroxi-4-metoxi-di-hidrochalcona;

$(2 S)$-Narigenina $(2 S)$-Eriodictiol; $(2 S)$-Liquiritigenina;

$(2 S)$-Liquiritigenina 7-metoxi; $(2 S)$-Liquiritigenina 4-metoxi;

(2S)-7,4-Di-hidroxiflavona; (2S)-7,3-Dimetoxi-4-hidroxi-flavona;

(2S)-3,4-Dimetoxi-7-hidroxi-flavona;

(2S)-7,4-Dimetoxi-3-hidroxi-flavona

Estilbenóides

Obtustireno

Outros 5,7-di-hidroxicromona; $(2 R, 3 R)$-3-O-galoilepicatequina

Glicina; Alanina; Valina; Metionina; Isoleucina; Leucina; Tirosina; 
Tabela 1. (continuação)

\begin{tabular}{lccc}
\hline B.racemosa & Cromanos & Pacharina; Racemosol; Des-O-metilracemosol \\
\hline B. reticulata & Flavonóides & Quercetina & 24 \\
\hline B. rufescens & Estilbenóides & 5,6-di-hidro-11-metoxi-2,2,12-trimetil-2H-nafto-[1,2-£][1]- & 26 \\
& & benzopirano-8,9-diol; 11 -metoxi-2,2,12-trimetil-2H-nafto-
\end{tabular}

[1,2-£][1]-benzopirano-8,9-diol; 1,7,8,12b-tetra-hidro-2,2,4-

trimetil-2H-benzo-[6,7]-ciclo-hepta-[1,2,3-de]

[1]benzopirano-5,10,11-triol

\begin{tabular}{|c|c|c|c|}
\hline \multirow[t]{6}{*}{ B.splendens } & Esteróides & Sitosterol; Estigmasterol & 27 \\
\hline & Ácidos graxos & Ácido esteárico & \multirow{5}{*}{28} \\
\hline & \multirow[t]{3}{*}{ Flavonóides } & Bausplendina & \\
\hline & & Quercetina & \\
\hline & & Rutina & \\
\hline & Benzenóide & Galato de etila & \\
\hline B. tomentosa & Flavonóides & Isoquercitrina; Quercetina; Rutina & 29,30 \\
\hline B. thonningii & Lactona & Grifonilida & 31 \\
\hline \multirow[t]{11}{*}{ B. uruguayensis } & \multirow[t]{5}{*}{ Esteróides } & Estigmasta-1,3,5-trieno; Estigmasta 3,5-dieno; Campesterol; & \multirow[t]{11}{*}{32} \\
\hline & & Estigmasterol; Sitosterol; Estigmasta-4,6-dien-3-ona; Sitosterol- & \\
\hline & & 3-O- $\alpha$-D-riburono-furanosídio; Sitosterol -3-O- $\beta$-D-xilopiranosídio; & \\
\hline & & Sitosterol-3- $O$ - $\alpha$-D-xiluronofuranosídio; & \\
\hline & & Sitosterol -3-O- $\beta$-D-glucopiranosídio & \\
\hline & \multirow[t]{2}{*}{ Flavonóides } & Quercetina -3-O- $\alpha$-L-ramnopiranosídio; & \\
\hline & & Kaempferol -3-O- $\alpha$-L-ramnopiranosídio & \\
\hline & \multirow[t]{3}{*}{ Aminoácidos } & Ácido aspártico; Treonina; Serina; Ácido glutamico; Prolina; & \\
\hline & & Glicina; Alanina; Valina; Metionina;Isoleucina; Leucina; Tirosina; & \\
\hline & & Fenilalanina; Histidina; Colina & \\
\hline & Outros & Hidrocarbonetos; Álcoois lineares & \\
\hline \multirow[t]{3}{*}{ B.vahlii } & Esteróides & Campesterol; Estigmasterol; Sitosterol & \multirow[t]{3}{*}{33} \\
\hline & Flavonóides & Quercetina; Quercetina-3-glucosídio; Kaempferol; Agathisflavona & \\
\hline & Triterpenóides & Ácido betulínico & \\
\hline \multirow[t]{2}{*}{ B. megalandra } & Flavonóides & 5,7,5'-tri-hidroxi-2'- $O$-ramnosil-flavona; $5,7,2^{\prime}$-tri- & \multirow[t]{2}{*}{34} \\
\hline & & hidroxi-5'-O-ramnosil-flavona & \\
\hline \multirow[t]{4}{*}{ B.variegata } & Esteróides & Sitosterol & \multirow[t]{2}{*}{35} \\
\hline & Triterpenóides & Lupeol & \\
\hline & \multirow[t]{2}{*}{ Flavonóides } & Narigenina-5,7-dimetoxi-4-ramnoglucosídio; & \multirow[t]{2}{*}{35,36} \\
\hline & & Kaempferol-3-galactosídio; Kaempferol-3-ramno-glucosídio & \\
\hline
\end{tabular}

fitoterápicas, uma vez que esta planta já está disponível no mercado farmacêutico. Muitos destes estudos são antigos, como o relatado por Carmela Julian ${ }^{42}$, ressaltando que o extrato de B. forficata deve ser administrado, não só no tratamento do diabetes, mas também nos distúrbios endócrinos, que contribuem para manifestar um esta$\mathrm{do}^{37,42,43}$. Russo e colaboradores ${ }^{44}$ demonstraram que a infusão preparada com as folhas da $B$. forficata não apresentou efeito hipoglicemiante em pacientes com glicemia normal e em pacientes com diabetes Tipo II (não-insulino dependente). Quando se analisou a ação hipoglicemiante do decocto da planta (150 g/L de água), imitando-se o uso na medicina popular, através da administração oral crônica em ratos diabéticos, os animais mostraram uma melhoria no metabolismo de carboidratos verificado pelos menores níveis de glicemia e glicosúria ${ }^{45}$. 
<smiles>C/C=C1/C=CC(OC)C(O)C1OC(O)C(CO)C(CO)CO</smiles><smiles>O=c1c(OC2OC3C(O)C(O)C(O)C(O)C3OC2O)c(-c2ccc(O)cc2)oc2cc(O)cc(O)c12</smiles><smiles>COc1cc2oc(-c3ccc4c(c3)OCO4)cc(=O)c2c2c1OCO2</smiles>

Bausplendina

5-di-hidroxi-7-metoxiflavona- 6-O- $\beta$-xilopiranosídio<smiles>COc1cc2c(c(O)c1C)Oc1cccc(O)c1C=C2</smiles><smiles>COc1ccc(/C=C/C(=O)c2ccc(O)cc2O)cc1</smiles>

Isoliquiritigenina-4-metoxi<smiles>COc1cc2oc(-c3ccccc3)cc(=O)c2c(O)c1OC</smiles><smiles>CCCOc1ccc2c(c1O)CCc1cc(O)c(C)c3c1C2CC(C)(C)C3</smiles>

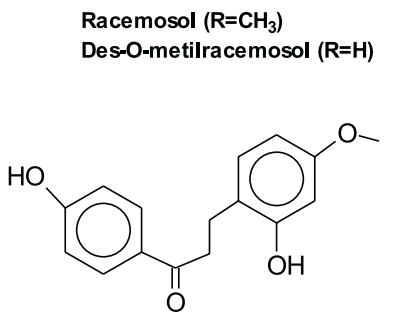

2,4'-di-hidróxi-4-metóxi-di-hidrochalcona

Por outro lado, em contraste com dados disponíveis na literatura sobre o efeito antidiabético de B. forficata, Damasceno e colaboradores $^{46}$ verificaram recentemente que o extrato alcoólico de folhas desta planta não reduz a concentração da glicose em ratos diabéticos induzidos pela streptozotocina.

Os resultados discrepantes podem ser devidos a vários aspectos não levados em consideração nestas investigações, como os fatores ambientais (tipo de solo, clima, etc) e sazonais ${ }^{2}$. O extrato metanólico da $B$. cheilantha apresentou efeito hipoglicemiante na dose de 600 $\mathrm{mg} / \mathrm{kg}$ quando se induziu o diabetes com o aloxano $(150 \mathrm{mg} / \mathrm{kg} \text {, i.p })^{47}$.

Os polifenóis (flavonóides) existentes no extrato hidroalcoólico $(1000 \mathrm{mg} / \mathrm{kg})$ das sementes da B. variegata mostraram efeito hipoglicemiante em ratos, nos quais o diabetes foi induzido pela streptozotocina $(55 \mathrm{mg} / \mathrm{kg})^{48}$.

A avaliação de B. monandra como possível agente hipoglicemiante através do método Glicose-Oxidase, permitiu observar que o extrato hidroalcoólico (500 mg/Kg, v.o.) desta planta apresenta ação hipoglicemiante após 4 h, maior que a insulina ${ }^{49}$. Recentemente, Minto e Pereira ${ }^{50}$ confirmaram a ação antidiabética desta planta, demonstrando que o chá das folhas secas $(10 \%)$ reduz significativamente o nível glicêmico em camundongos, cujo efeito é observado até $6 \mathrm{~h}$ após a administração do chá.

O extrato aquoso obtido de folhas de $B$. megalandra causou ini- bição da glicose-6-fosfatase, enzima que pode ser de utilidade no controle da hiperglicemia presente no diabético ${ }^{51}$. Este efeito parece estar relacionado, pelo menos parcialmente, com a presença dos flavonóides indicados na Tabela $1^{34}$.

\section{Atividade antimicrobiana das plantas e compostos isolados do gênero Bauhinia}

A espécie $B$. splendens, utilizada no Brasil como um agente antiinfeccioso, foi investigada quanto à possível atividade antibacteriana através do método da difusão em ágar e pela determinação da concentração inibitória mínima (CIM), mostrando-se ativa contra bactérias Gram-positivas e Gram-negativas. Os extratos de diclorometano (DCM) e acetato de etila (AE) das folhas desta planta foram ativos no modelo de difusão em ágar contra Salmonella sp. em todas as concentrações utilizadas (1,25-3,75 mg). O extrato de DCM também mostrou-se ativo contra Streptococcus sp. e Staphylococcus aureus, com uma zona de inibição de 26 e $18 \mathrm{~mm}$, respectivamente, na concentração de $2,5 \mathrm{mg} /$ disco. Por outro lado, o extrato aquoso desta planta não apresentou atividade antibacteriana.

Em relação à determinação da Concentração Inibitória Mínima (CIM), o extrato de DCM apresentou valores de $0,7 \mathrm{mg} / \mathrm{ml}$ contra $S$. typhimurium e $0,4 \mathrm{mg} / \mathrm{ml}$ contra $S$. aureus, e o extrato de AE apre- 
sentou CIM de $0,8 \mathrm{mg} / \mathrm{ml}$ e $0,4 \mathrm{mg} / \mathrm{ml}$ respectivamente. Estes efeitos parecem estar relacionados com os compostos fenólicos (rutina, quercetina e galato de etila) isolados desta planta ${ }^{28,52}$.

Compostos isolados da B. manca apresentaram significativa atividade antifúngica contra as espécies Botrytis cinerea, Claviceps viridis, Coprinus cinereus, Rhizoctonia solani e Saprolegnia asterophora. Os compostos com maior atividade foram (2S)-7,4-dihidroxiflavona, $(2 S)$-4-hidroxi-7-metoxiflavona, $(2 S)$-7,3-dimetoxi4-hidroxiflavona e (2S)-4-acetoxi-7,3-dimetoxiflavona ${ }^{11}$.

O extrato de DCM, obtido a partir de cascas de B. rufescens, apresentou atividade antifúngica contra a espécie Cladosporium cucumerinum. Os estudos fitoquímicos com esta planta permitiram o isolamento de quatro novos compostos tetracíclicos antifúngicos, identificados como 5,6-di-hidro-11-metoxi-2,2,12-trimetil-2H-nafto[1,2-£][1]-benzopirano-8,9-diol; 11-metoxi-2,2,12-trimetil-2H-nafto[1,2-£][1]-benzopirano-8,9-diol; 1,7,8,12b-tetra-hidro-2,2,4-trimetil2H-benzo-[6,7]-ciclo-hepta-[1,2,3-de][1]-benzopirano-5,9,10-triol; 1,7,8,12b-tetra-hidro-2,2,4-trimetil-2H-benzo-[6,7]-ciclo-hepta[1,2,3-de][1] benzo- pirano-5,10,11-triol ${ }^{26}$.

Em um screening de plantas usadas tradicionalmente no Nepal para tratar de doenças que podem ser causadas por vírus, os extratos metanólicos de algumas espécies de Bauhinia mostraram-se parcialmente eficazes contra os microorganismos Herpes simplex, Sindbis virus e Poliovirus ${ }^{53}$.

Os extratos de hexano (HEX), DCM e AE obtidos de folhas, caules e cascas de $B$. forficata foram testados contra fungos e leveduras patogênicos. Para a realização dos testes antifúngicos através da diluição em ágar, concentrações de até $1000 \mu \mathrm{g} / \mathrm{ml}$ foram incorporados nos meios de crescimento. Os resultados mostraram que nenhum dos extratos testados apresenta atividade contra leveduras (Candida albicans, Candida tropicalis, Saccharomyces cerevisiae, Cryptococcus neoformans, Aspergillus flavus, Aspergillus fumigatis, Aspergillus niger) cujas CIMs foram acima de $1000 \mu \mathrm{g} / \mathrm{ml}$. Por outro lado, diferentes resultados foram obtidos nos testes dos extratos de $B$. forficata contra dermatófitos ${ }^{54}$. O extrato de $\mathrm{AE}$ dos caules mostrou-se moderadamente ativo contra Epidermophyton floccosum, com CIM de $750 \mu \mathrm{g} / \mathrm{ml}$, assim como os extratos de HEX das cascas, o qual foi ativo contra M. canis, T. mentagrophytes, T. rubrum e E. floccosum com $\mathrm{CIM}<1000 \mathrm{mg} / \mathrm{ml}$ para todas as espécies fúngicas estudadas. O extrato de DCM das cascas apresentou o melhor resultado antifúngico, sendo ativo contra T. rubrum e E. floccosum com CIM de $500 \mu \mathrm{g} / \mathrm{ml}^{54}$.

Souza e colaboradores ${ }^{55}$ avaliaram a atividade antimicrobiana dos extratos e frações da $B$. forficata e B. microstachya através do método de difusão radial em ágar, observando que somente uma fração da $B$. forficata inibiu o crescimento da $E$. coli $e$ S. aureus na concentração de $1000 \mu \mathrm{g} / \mathrm{ml}$. A espécie $B$. microstachya não apresentou atividade antimicrobiana.

\section{Outras atividades farmacológicas apresentadas pelas plantas do gênero Bauhinia}

Inibidores de tripsina foram purificados do extrato salino de sementes de $B$. bauhinioides através de uma coluna cromatográfica de troca iônica. Dessa planta foram isolados os inibidores BbTI-I e BbTIII. Este último, inibe a kalicreína pancreática, hidrolisando os aminoácidos H-Pro-Phe-Arg-ACM ${ }^{56,57}$.

A espécie $B$. forficata foi avaliada através do teste geral de atividades (TGA), sendo que observou-se uma intensa diurese após dez minutos de administração do extrato bruto da tintura, denotando sua ação diurética. Com relação aos efeitos sobre a musculatura lisa e S.N.C., concluiu-se que somente em doses acima de $2,7 \mathrm{~g} / \mathrm{kg}$, evidenciam-se tais efeitos. Quanto à toxicidade aguda, observou-se que o extrato bruto da planta provocou morte em 50\% dos animais na dose de 2,85 g/kg, quando administrado por via intraperitoneal. Nas doses de 0,5 a $5,0 \mathrm{~g} / \mathrm{kg}$ administrados por via oral, o extrato bruto não apresentou qualquer efeito tóxico. A atividade antiedematogênica, associada à ação analgésica periférica, demonstra fortes indícios de que o extrato bruto de B. forficata possui ação antiinflamatória, porém estes resultados ainda necessitam de estudos complementares ${ }^{58,59}$.

Carvalho e colaboradores ${ }^{60}$ demonstraram que alguns extratos obtidos da casca do caule de B. guianensis exercem atividade antiinflamatória no modelo de edema de pata de rato, induzido pela carragenina, dextrana e histamina, além de efeito analgésico no modelo de contorções abdominais induzidas pela ácido acético em camundongos.

Os extratos hidroalcoólicos e de acetato de etila $(10 \mathrm{mg} / \mathrm{kg})$ das folhas, caules, cascas e raízes de $B$. splendens apresentaram considerável efeito analgésico quando analisados no modelo de dor causado pelo ácido acético $0,6 \%$ em camundongos, sendo mais eficazes do que a aspirina e o paracetamol, dois fármacos amplamente utilizados na clínica ${ }^{28}$

O extrato hidroalcóolico de B. splendens, na dose de 3-60 mg/kg (i.p) ou $50-400 \mathrm{mg} / \mathrm{kg}$ (oral) causou inibição dose-dependente na constricção abdominal causada pelo ácido acético, com $\mathrm{DI}_{50}$ de $3 \mathrm{mg} / \mathrm{kg}$ (via i.p) e $177,6 \mathrm{mg} / \mathrm{kg}$ (via oral) e inibição máxima de $95 \pm$ 2 e $61 \pm 6 \%$, respectivamente. No teste da formalina, na dose de 1 $60 \mathrm{mg} / \mathrm{kg}$ (i.p.) e 50-400 mg/kg (oral), a espécie B. splendens mostrou-se mais ativa na segunda fase da dor induzida pela formalina. As porcentagens de inibição máxima para a primeira e segunda fase da dor foram $68 \pm 6$ e $99 \pm 1 \%$, respectivamente, pela via i.p. e $37 \pm$ 6 e $69 \pm 9 \%$, pela via oral. Porém, estas mesmas doses não foram significativamente eficazes na resposta edematogênica causada pela formalina. O mecanismo de ação do extrato de $B$. splendens ainda continua desconhecido, porém parece estar relacionado com a modulação de receptores opióides ${ }^{61}$.

Schmeling e colaboradores ${ }^{62}$ avaliaram o efeito antinociceptivo e anti-hiperalgésico do extrato hidroalcoólico obtido de partes aéreas de B. microstachya nos modelos de dor induzida pela injeção intraplantar de capsaicina (CAP) e intraperitoneal de ácido acético em camundongos e na hiperalgesia induzida pela injeção intraplantar de diversos agentes flogísticos, como a carragenina (CAR), bradicinina (BK), capsaicina (CAP), substância P (SP) e adrenalina (ADR). O extrato obtido de B. microstachya apresentou significativo efeito antinociceptivo quando analisado na nocicepção química induzida pela capsaicina e pelo ácido acético em camundongos. Além disso, o extrato desta planta também apresentou importante redução da hiperalgesia induzida por CAR, BK, CAP, SP e ADR em ratos, substâncias estas envolvidas na transmissão da dor.

O extrato hidroalcoólico obtido a partir da B. variegata apresentou inibição não-competitiva na contração da musculatura lisa de útero de $\operatorname{ratas}^{48}$.

A administração diária do extrato das cascas de B. purpurea $(2,5$ $\mathrm{mg} / \mathrm{kg}$ i.p.) em ratas com disfunção tiroidiana revelou que esta planta é capaz de aumentar a atividade da glicose-6-fosfatase, assim como apresentou efeitos antiperoxidativos, indicado pela diminuição da peroxidação lipídica hepática e aumento da atividade enzimática antioxidante. Assim, concluiu-se que esta planta é capaz de estimular a função tiroidiana de $\operatorname{ratas}^{63}$.

A atividade antiulcerogênica do extrato metanólico de flores de B. racemosa foi estudado em ratos através da indução de úlceras gástricas pela aspirina, as quais foram significativamente reduzidas pelo emprego do extrato desta planta ${ }^{64}$.

Trinta espécies de plantas tradicionalmente usadas na Bolívia contra os males da malária tiveram recentemente seus extratos testados frente à atividade antimalárica. Dentre estas espécies destaca-se a B. guianensis, a qual apresentou bons resultados, atuando princi- 
palmente para amenizar os sintomas associados a esta patologia ${ }^{65}$.

Estudos recentes indicaram que a $B$. tarapotensis apresenta várias substâncias com perfil antioxidante ${ }^{66}$.

\section{CONCLUSÕES E PERSPECTIVAS}

O uso medicinal das plantas pertencentes ao gênero Bauhinia pela população de diferentes partes do mundo tem encontrado respaldo nos estudos científicos, que comprovam a eficácia destas plantas em vários modelos experimentais. Neste contexto, alguns efeitos biológicos ou farmacológicos, como antifúngicos, antibacterianos, analgésicos, antiinflamatórios e especialmente antidiabéticos, são relatados na literatura, comprovando e justificando o uso destas espécies na medicina popular.

Embora muitos compostos, incluindo alcalóides, terpenos, esteróides, estilbenos, etc, mas principalmente flavonóides, tenham sido isolados e identificados nestas espécies, são raros os estudos que relacionam tais compostos aos efeitos biológicos indicados.

Cabe ressaltar, por exemplo, que a maioria dos compostos ativos como hipoglicêmicos continuam obscuros e a descoberta destas substâncias poderia levar à obtenção de novos fitofármacos ou mesmo fármacos para o tratamento de diabetes do tipo II, patologia que aflige um grande número de pessoas em todo o mundo.

\section{AGRADECIMENTOS}

Os autores agradecem ao CNPq, à ProPPEX e ao CCS/UNIVALI e à Fundação Herbarium de Saúde e Pesquisa pelo auxílio financeiro no desenvolvimento de projetos realizados com algumas espécies de plantas do gênero Bauhinia.

\section{REFERÊNCIAS}

1. Ugaz, O. L.; Investigación Fitoquímica; Fondo Editorial. Lima: Pontificia Universidad Católica del Peru, Lima: Peru, 1994.

2. Cechinel Filho, V.; Yunes, R. A.; Quim. Nova 1998, 21, 99.

3. Hamburger, M.; Hostettmann, K.; Phytochemistry 1991, 30, 3864.

4. Mitscher, L. A.; Drake, S.; Gollapudi, S.R.; Okunte, K. A.; J. Nat. Prod. 1987, 50, 1025 .

5. Cechinel Filho, V.; Dal Magro, J.; Yunes, R. A.; Grifos 1996, 3, 63

6. Yunes, R.A.; Pedrosa, R.C.; Cechinel Filho, V.; Quim. Nova 2001, 24, 147.

7. Cechinel Filho, V.; Quim. Nova 2000, 23, 680.

8. Lozoya, X.; Investigación y Ciência 1997, Nov, 4.

9. Yamada, C.S.B.; Racine 1998, 2, 50.

10. Cragg, G. M.; Newman, D. J.; Snader, K.M.; J. Nat. Prod. 1997, 60, 52.

11. Achenbach, H.; Stocker, M.; Constenla, M. A. F.; Phytochemistry 1988, 27, 1835.

12. Breviglieri, E; Monografia de Conclusão de Curso, Universidade do Vale do Itajaí, Brasil, 1997.

13. Teske, M; Trentini, A..M. M.; Compêndio de Fitoterapia; Herbarium Lab. Botânico, Curitiba: Paraná, 1995.

14. Gupta, M.P., ed.; 270 Plantas Medicinales Iberoamericanas; Talleres de Editorial Presencia, Santafé de Bogotá: Colombia, 1995, p. 347.

15. Iribarren, A. M.; Pomilio, A. B.; J. Nat. Prod. 1983, 46, 752.

16. Iribarren, A. M.; Pomilio, A. B.; Phytochemistry 1987, 26, 857.

17. Chien-Chin, C.; Yuh-Pan, C.; Hong-Yen, H. B.; J. Nat. Prod. 1985, 48, 933.

18. Silva, K.L.; Biavatti, M.W.; Leite, S.N.; Yunes, R. A.; Delle Monache, F.; Cechinel Filho, V.; Z. Naturforsch. 2000, 55, 478.

19. Sousa, E.; Szpoganicz, B.; Pizzolatti, M.G.; VI Encontro de Química da Região Sul da Sociedade Brasileira de Química, Maringá, Brasil, 1998.

20. Viana, E. P.; Santa Rosa, R.S.; Almeida, S.S.M.S.; Santos, L.S.; Resumos da $23^{a}$ Reunião Anual da Sociedade Brasileira de Química, Caxambu, Brasil, 2000.

21. Ramachandran, R.; Joshi, B.C.; Curr. Sci. 1967, 21, 574.

22. Vijayakumari, K.; Siddhuraju, P.; Janardhanan, K. L.; J. Sci. Agric. 1997, 7, 279.

23. Yadava, R.N.; Tripathi, P.; Fitoterapia 2000, 71, 88.

24. Prabhakar, P.; Gandhidasan, R.; Raman, P.V.; Krishnasamy, N.R.; Nanduri, S.; Phytochemistry 1994, 36, 817.
25. Rabate, J.; J. Pharm. Chin. 1938, 28, 435.

26. Maillard, M.P.; Recio Iglesias, M.C.; Saadou, M.; Stoelkli Evans, H.; Hostettmann, K.; Helv. Chim. Acta 1991, 74, 791.

27. Laux, D. O.; Stefan, G.M.; Gottlieb, O. R.; Phytochemistry 1985, 24,1081.

28. Cechinel Filho, V.; Breviglieri, E.; Willain Filho, A.; Santos A.R.S.; Rev. Bras. Farm. 1995, 76, 115.

29. Subramanian, S.S.; Nair, A.G.R.; J. Chem. 1963, 1, 450.

30. Row, L.; Ramachandra, L.; Viswanadham, N.; Proc. Indian. Acad. Sci. 1954, 39, 240.

31. Okwute, S. K.; Ndukwe, G.I.; J. Nat. Prod. 1986, 49, 716.

32. Iribarren, A. M.; Pomilio, A. B.; Assoc. Quim. Argent. 1989,77, 461.

33. Sultana, S.; Ilyas, M.; Mohammed, K.; Shaida, W.A.; J. Indian Chem. Soc. $1985,8,337$.

34. Gonzalez-Mujica, F.; Motta, N.; Perdomo, E.; Méndez, J.; Hasegawa, J.; Rev. Latinoamer. Quim. 2000, 28, 127.

35. Gupta, A. K.; Vidypat, T.J.; Chauhan, J.S.; Planta Med. 1980, 38, 174.

36. Rahman, W.; Begun, S.J.; Naturwissenchaften 1966, 53, 385.

37. Oliveira, F.; Saito, M.L.; Rev. Bras. Farmacog. 1987/1989, 2-4, 170.

38. Ramos, R.R.; Alarcon-Aguilar, F.; Lara-Lemus, A.; Flores-Saenz, J.L.; Arch. Med. Res. 1992, 23, 59.

39. Lemus, I.; Garcia, R.; Delvillar, E.; Knop, G.; Phytother. Res. 1999, 13, 91.

40. Shani, J.; Goldschmied, A.; Joseph, B.; Ahronson, Z.; Sulman, F.G.; Arch. Int. Pharmacodyn. Ther. 1974, 210, 27.

41. Weiczel, G.; Waahl, P.; Buddecke, E.; Physiol. Chem. 1962, 327, 109.

42. Julian, C.; Rev. Med. Pharm. Chem. Phys. 1929, $2,1$.

43. Costa, O. A. ; Leandra 1975, 5, 104.

44. Russo, E.M.K.; Reichelt, A.A.J.; De-Sá, J.R.; Furlanetto, R.P.; Moisés, R.C.; Kasamatsu, T.S.; Chacra, A.R.; Braz. J. Med. Biol. Res. 1990, 23, 11.

45. Pepato, M.T.; Keller, E.H.; Baviera, A.M.; Silva, M.P.M.; Vendramini, R.C.; Bruneti, I.L. XIII Reunião Anual da FSBE, Caxambú, Brasil, 1998.

46. Damasceno, D. C.; Volpato, G. T.; Sartori, T. C. F.; Rodrigues, P. F.; Perin, E. A.; Calderon, I. M. P.; Rudge, M. V. C.; XVI Latinoamerican Congress of Pharmacology, Águas de Lindóia, Brasil, 2000.

47. Almeida, E. R.; Xavier, H.; Guedes, M. C.; III Jornada Paulista de Plantas Medicinais, Campinas, Brasil, 1997.

48. Wazlawik, E.; Silva, M. A.; Peters, R.R.; Simões, C.M.O.; Ribeiro-Do-Vale, R.M.; IX Reunião Anual da FSBE, Caxambú, Brasil, 1994.

49. Anjos, F.B.R; Canto Jr. A.S.; Leôncio, M.P.; Santos, R.V.H.; Afiatpour, P.; IX Reunião Anual da FSBE, Caxambú, Brasil, 1994.

50. Minto, A. B. M.; Pereira, M. A.; XVI Latinoamerican Congress of Pharmacology, Águas de Lindóia, Brasil, 2000.

51. Gonzalez-Mujica, F.; Motta, N.; Becerra, A.; Phytother. Res. 1998, 12, 291.

52. Savi, A.O.S.; Breviglieri, E.; Bella Cruz, A.; Yunes, R. A.; Cechinel Filho, V.; Rev. Biol. Trop. 1997, 44, 601.

53. Taylor, R.S.; Hudson, J.B.; Manandhar, N.P.; Towers, G.H.; J. Ethnopharmacol. 1996, 53, 97.

54. Silva, K.L.; Monografia de Conclusão de Curso, Universidade do Vale do Itajaí, Brasil, 1999.

55. Souza, R. S. S.; Santos, D. R.; Bella Cruz, R. C.; VI Seminário Integrado de Iniciação Científica, Camboriú, Brasil, 2000.

56. Oliva, M.L.; Mendes, C.R.; Juliano, M.; Chagas J. R.; Rosa, J. C.; Greene, L.J.; Sampaio, M. U.; Sampaio, C. A.; Immunopharmacology 1999, 45, 163

57. Oliva, M.L.; Andrade, S. L.; Batista, I. F.; Sampaio, M. U.; Juliano, M.; Fritz, H.; Auerswald, E. A.; Sampaio, C. A.; Immunopharmacology 1999, $45,145$.

58. Luz, M. M. S.; Monografia de Especialização em Ciências Farmacêuticas, Universidade Federal do Paraná, Curitiba, Brasil, 1995.

59. Luz, M.M.S.; Santos, C.A.M.; Sato, M.E.O.; Arruda, A.M.S.; XIV Simpósio de Plantas Medicinais do Brasil, Florianópolis, Brasil, 1996.

60. Carvalho, J.C.T.; Santos, L.S.; Viana, E.P.; de Almeida, S.S.M.S.; Marconato, E.; Rodrigues, M.; Ferreira, L.R.; Van de Kamp, A.; Pharmaceut. Biol. 1999, 37, 281.

61. Willain Filho, A.; Breviglieri, E.; Cechinel Filho, V.; Santos A.R.S.; J. Pharm. Pharmacol. 1997, 49, 823.

62. Schmeling, L. O.; Gadotti, V. M.; Liz, F. H.; Machado, C.; Meyre-Silva, C.; Santos, A. R. S.; Cechinel Filho, V.; VI Seminário Integrado de Iniciação Científica, Camboriú, Brasil, 2000.

63. Panda, S; Kar, A.; J. Ethnopharmacol. 1999, 67, 233.

64. Akhtar, A.H.; Ahmad, K.U.; J. Ethnopharmacol. 1995, 46, 1.

65. Munoz,V.; Sauvain, M.; Bourdy, G.; Bergeron, S.; Rojas, I.; Bravo, J.A.; Balderrama, L.; Ortiz, B.; Gimenez, A.; Deharo, E.; J. Ethnopharmacol. 2000, 69, 127

66. Braca, A.; Tommasi, N.; Di Bari, L.; Pizza, C.; Politi, M.; Morelli, I.; J. Nat. Prod. 2001, 64, 892. 